\title{
SLC17A2 Expression Correlates with Prognosis and Immune Infiltrates in Hepatocellular Carcinoma
}

\section{Zhijian Wang}

The First Affiliated Hospital of Chongqing Medical University

\section{Xuenuo Chen}

The First Affiliated Hospital of Chongqing Medical University

Zheng Jiang ( $\square$ zhengj1753@126.com )

The First Affiliated Hospital of Chongqing Medical University

\section{Research Article}

Keywords: SLC17A2, hepatocellular carcinoma (HCC), independent prognostic factor, bioinformatics, immune cell infiltration

Posted Date: June 7th, 2021

DOI: https://doi.org/10.21203/rs.3.rs-573999/v1

License: (c) (1) This work is licensed under a Creative Commons Attribution 4.0 International License. Read Full License 


\section{Abstract}

Background: Hepatocellular carcinoma (HCC) is one of the most common malignant tumors with a dismal prognosis according to updated statistics. At present, there are many deficiencies in targeted therapy for liver cancer. The solute carrier family 17 member 2 (SLC17A2) has not been studied in liver cancer, therefore, we evaluate the role of SLC17A2 in HCC by bioinformatics analysis.

Methods: The expression level of SLC17A2 in HCC, the clinicopathological data were analyzed based on The Cancer Genome Atlas (TCGA) and Gene Expression Omnibus (GEO) database, the SLC17A2 protein expression was validated by immunohistochemical staining. Besides, the Kaplan-Meier plotter database and receiver operating characteristic (ROC) curve analysis were used to explore the prognostic significance. The biological analyses of SLC17A2 were performed using the gene set enrichment analysis (GSEA). Finally, Tumor Immune Estimation Resource (TIMER) and Gene Expression Profiling Interactive Analysis (GEPIA) databases were used to explore the relationship between immune cell infiltration, immune cell markers and SLC17A2 in HCC.

Results: The multivariate Cox regression analysis showed that SLC17A2 expression was low in $\mathrm{HCC}(\mathrm{P}<$ 0.05), and closely related to the clinical stage of HCC. Besides, SLC17A2 had certain prognostic and diagnostic value in $\mathrm{HCC}$ according to ROC curve analysis. Further biological analyses showed that SLC17A2 can regulate fatty acid metabolism, amino acid metabolism and cytochrome P450-related metabolism and is closely related to the peroxisome pathway. Notably, we found that SLC17A2 expression was positively correlated with the infiltration levels of $C D 4+T$ cells, naive $C D 8+T$ cells, naive $B$ cells, negatively associated with the levels of regulatory $T$ cells and closely correlated with most immune cell markers in HCC.

Conclusion: SLC17A2 expression is low in HCC and correlates with immune infiltration; thus, it could serve as an independent prognostic factor for HCC.

\section{Introduction}

According to the latest epidemiological statistics related to liver cancer, the incidence rate of hepatocellular carcinoma (HCC) ranks sixth in the world, and HCC is the fourth most common cause of cancer-related death. In 2015, 326000 patients died of liver cancer in China, and this number is on the rise. In addition, the incidence and mortality of liver cancer in rural residents were higher than those in city residents. The incidence and mortality were closely related to the basis of hepatitis $B$ virus infection, and the patients were not followed up[1]; moreover, fatty liver, alcohol-related cirrhosis, aflatoxin and a family history could not be excluded[2]. In clinical practice, for patients with early-stage tumors, radical resection, radiofrequency ablation and liver transplantation can be considered curative treatments. In addition, chemotherapy, radiotherapy, immunotherapy and targeted therapy can be applied as palliative treatments for intermediate and advanced cases. Nevertheless, the prognosis of HCC patients is poor, limiting the therapeutic effect. At present, the TNM staging system is widely used to evaluate the prognosis of 
patients, but the T stage of this system is controversial according to many surgeons. Gaya Spolverato et al.'s studies showed that the mortality risk of patients with T3 disease was lower than that of patients with T1b and T2 disease[3]. San Hyun Kang et al. showed no significant difference between patients with T2 and T3 disease[4]. In addition,although alpha fetoprotein (AFP) is used to evaluate diagnosis and prognosis, its sensitivity and specificity are unsatisfactory. Thus, a new independent prognostic and diagnostic factor that can be used to screen HCC early and guide clinical management is urgently needed.

The solute carrier (SLC) family of membrane transporters is high expressed in the liver on cell membranes and organelles[5, 6], which is the key to regulating transmembrane transport. Some studies shown that SLC uniporters meditate numerous essential physiological functions and cause diverse disease, for instance, neoplasm and metabolic disease. A recent study founded that SLC regulates lymphocyte signaling and differentiation by adjusting different metabolic pathways[7]. Members of the SLC family play an essential role in the development of various tumors. For example, SLC22A3 has been shown to be a proto-oncogene in colorectal cancer[8]. In addition, overexpression of the SLC3A2 protein is associated with a worse prognosis in breast cancer patients[9]. Furthermore, SLC38A3 serves as a prognostic factor and predictor of differential drug sensitivities in esophageal squamous cell carcinoma[10].

SLC17A2 is a protein-coding gene that is perceived only in the membrane and cytoplasm of hepatocyte, as shown in the tissue distribution diagram. One study indicated significant differentially methylated CpG sites between aggressive and indolent prostate cancers, and SLC17A2 was hypomethylated[11], which illustrated that the methylation of SLC17A2 enhanced tumor invasiveness. In addition, SLC17A2 is involved in various metabolic pathways as a critical transcription factor. For instance, one fresh fasting insulin locus at SLC17A2 was recently uncovered by a Metabochip-wide analysis[12], and we deduced that SLC17A2 may be related to glucose metabolism in tumor cells. However, the expression level of SLC17A2 and its relationship with prognosis in HCC remain unclear. The aim of our research was to evaluate the expression pattern and possible regulatory mechanisms of SLC17A2 in hepatocellular carcinoma, as it may become a valuable diagnostic and prognostic biomarker for liver cancer in the future. The research process is shown in Fig. 1.

\section{Materials And Methods}

Expression data of SLC17A2 extracted from The Cancer Genome Atlas (TCGA) and Gene Expression Omnibus (GEO):

We used the key word "liver cancer" to search the GEO database and regarded "Homo sapiens" as a restrictive condition. The microarray dataset GSE45267 (from the GPL570 platform), which includes 46 primary HCC samples and 41 paracancerous tissues from 61 patients, was acquired, and the data were preprocessed with the "sva" and "limma" packages in R software (version 4.0.4). In addition, the clinical pathology and SLC17A2 expression data were downloaded from the TCGA (https://portal.gdc. cancer. gov/) and verified in the GEO database. 


\section{Patient data and tissue samples:}

We collected 60 cases liver tissue sections from the Department of Pathology of Chongqing Medical University for immunohistochemistry. These samples were from 32 patients hospitalized from January 2019 to December 2020. None of these patients received chemotherapy, radiotherapy or immunotherapy before surgery. The study was approved by the ethics committee of the First Affiliated Hospital of Chongqing Medical University, and 32 patients signed informed consent forms.

\section{Immunohistochemistry:}

First, the pathological sections of liver cancer tissue samples were placed in a $60^{\circ} \mathrm{C}$ incubator for 2 hours. Then, the paraffin sections were dewaxed in xylene and rehydrated in ethanol with different concentration gradients. Then, the tissue sections were put into antigen repair solution containing citrate for antigen repair. Then, the slides were incubated in $3 \%$ hydrogen peroxide at room temperature and in the dark for 10 minutes to block the activity of endogenous peroxidase. After washing with PBS, the slides were sealed with goat serum working solution at room temperature and in the dark for 10 minutes. Then, the rabbit monoclonal antibody SLC17A2 (anti-SLC17A2 antibody; 1:400 dilution) was added to the tissue sections, and the sections were placed in a humid chamber at $4^{\circ} \mathrm{C}$ for 12 hours. The first antibody was washed out with PBS and incubated with the second antibody in a $37^{\circ} \mathrm{C}$ incubator for 15 minutes. Then, horseradish enzyme-labeled streptavidin working solution was added and incubated at $37^{\circ} \mathrm{C}$ for 15 minutes. Next, the sections were stained with DAB for 8 minutes, stained with hematoxylin for approximately 15 seconds, dehydrated and sealed with wax. Finally, the expression level of SLC17A2 was evaluated according to the semiquantitative immunoreactivity scoring system by two independent pathologists who did not know the basic information of the patients. The patients were divided into a high expression group and a low expression group.

\section{Main databases and application methods:}

Oncomine database analysis: Oncomine is the largest oncogene microarray database and integrated data mining platform in the world. The expression of SLC17A2 was explored using the Oncomine database (https://www.oncomine.org/) with the following filters: P-value $<0.0001$, fold change $=2$, and gene rank $=$ top $10 \%$.

UALCAN database analysis: UALCAN (http://UALCAN.path.uab.edu) offers convenient methods to obtain the TCGA data, perform pan-cancer gene analysis and so on.

Gene Expression Profiling Interactive Analysis (GEPIA) database analysis: The GEPIA database (http://gepia.cancer-pku.cn/) is a public database newly produced by the Chinese for cancer and normal gene expression profiling. RNA sequencing data from 9736 tumor samples and 8587 normal samples were obtained from the TCGA project and used to calculate the survival rate of HCC patients, the results are expressed as the overall survival (OS) rate. In addition, the relevance between the expression level of SLC17A2 and markers of immunocyte was explored. 
c-BioPortal database analysis: The DNA mutation data and mRNA expression data were obtained through the cBioPortal for Cancer Genomics (https:// www.cbioportal. org/). This portal was also used to search for coexpressed genes to promote the exploration of multidimensional cancer gene data.

Gene Set Enrichment Analysis (GSEA) database analysis: The expression levels of SLC17A2 in samples from the TCGA LIHC RNA- Seq dataset were grouped according to the average SLC17A2 expression level: the top $50 \%$ of samples were classified as the high expression group, and the bottom $50 \%$ of samples were classified as the low expression group. Then, GSEA (version 4.0 http://software.broadinstitute.org/gsea/downloads.jsp) was used to analyze the effect of the SLC17A2 expression level on the gene set of each biological pathway. The screening standards used were as follows: P囚0. 05, FDR囚0. 25, and ES囚0.6.

STRING database and Cytoscape analyses: The STRING database (www.string-db.org) was used to predict the protein-protein interaction (PPI) network and to mine the core regulatory genes. After downloading the relevant data, we used Cytoscape software (Version3.8.2) to display the network. CytoHubba is a plugin of Cytoscape that can be used to extract the hub genes. In the network, each node represents a kind of protein, and the corresponding gene is marked on the node.

TIMER database analysis: TIMER (http://timer. cistrome.org/) is a comprehensive database comprising 32 cancer types from the TCGA that is used to analyze the infiltration of immune cells, mostly B cells, CD4 + T cells, CD8 + T cells, regulatory T cells (Tregs), neutrophils, macrophages and dendritic cells, in tumor tissues.

\section{Statistical methods:}

IBM Statistical Product and Service Solutions Statistics 25.0 and GraphPad Prism 7.0 software were used to input, organize and analyze the data. A Cox regression model and Student's t test were used to compare the differences between the groups, and a $\mathrm{P}<0.05$ was considered to indicate statistical significance. Furthermore, the prognostic value of SLC17A2 was evaluated by survival analysis, and its diagnostic value was assessed by receiver operating characteristic (ROC) Curve analysis. Exploring the correlations between the SLC17A2 expression and immune cells makers and to control for tumor purity at the same time using Pearson method. $\mathrm{P}<0.05$ indicated a significant correlation.

\section{Results}

Low expression of SLC17A2 in HCC. Firstly, we aimed to determine the distinctions in SLC17A2 expression between normal and tumor tissues of pan-cancer. The TIMER "DiffExp" module revealed that SLC17A2 expression was relatively higher in renal clear cell carcinoma and renal papillary cell carcinoma. In addition, SLC17A2 expression was notably lower in cholangiocarcinoma, renal chromophobe cell cancer and liver HCC tissues than in normal tissues ( $p<0.001$, Fig. $2 A)$.

In addition, we also used the Oncomine database to explore the differential expression of SLC17A2 in 7 studies, and found that SLC17A2 expression was significantly lower in HCC tissues than in normal 
tissues (Fig. 2B).

Furthermore, 377 patients in the TCGA database were classified according to the following clinical pathology factors: age, sex, pathological stage and grade, survival time and disease state. The TCGA data showed that the expression of SLC17A2 was remarkably lower in HCC tissues than in paracancerous tissues (Fig. $2 \mathrm{C}, \mathrm{P}<0.001$ ). Then GEO datasets from the National Center for Biotechnology Information (NCBI) were retrieved, and the RNA-seq data of SLC17A2 were obtained from the GSE45267 dataset, which collates 87 gene expression profiles of tissue samples from 61 patients: 46 primary HCC tissues and 41 paracancerous tissues. A t-test was performed on the GEO data and a boxplot was drawn (Fig. 2D, P < 0.0001). In addition, two other databases obtained from the GEO, GSE14520 (Fig. 2E, P < 0.0001) and GSE54236 (Fig. 2F, P < 0.01), were used for further verification. In conclusion, all the results confirmed that SLC17A2 was expressed at low levels in HCC.

Validation of SLC17A2 protein expression by immunohistochemical staining. The expression level of SLC17A2 protein in the cancer tissues and adjacent normal tissues of 32 patients with liver cancer was verified by immunohistochemistry (Fig. 3). The results showed that the expression level of SLC17A2 in cancer tissue was significantly lower than that in adjacent tissues $(n=60, P<0.0001$, Table I). In conclusion, all the results confirmed that SLC17A2 was expressed at low levels in HCC.

In addition, we mined cBioPortal data, which revealed that SLC17A2 expression was altered in 4\% (16/400) of samples, including 14 cases of amplification, and 2 cases of a missense mutation (Fig. 4A). In summary, amplification is the most common type of SLC17A2 mutation in HCC.

Prognostic significance of SLC17A2 in HCC patients. By mining data in the TCGA and generating a Kaplan-Meier survival curve, which collated 344 patients to investigate the relationship between SLC17A2 expression and the prognosis of HCC patients, we concluded that patients with higher SLC17A2 mRNA expression had longer overall survival times than those with lower SLC17A2 mRNA expression ( $\mathrm{P}=$ 0.0078, Fig. 4B). The same conclusion was draw by analyzing data from OncoLnc $(P<0.05$, Fig. $4 \mathrm{C})$ and GEPIA ( $P=0.0059$, Fig. 4D). In addition, Cox regression analysis was performed on the subgroups according to clinical-pathology factors, and the univariate Cox analysis indicated that SLC17A2, T-stage 3-4, M stage and TNM stage were significant factors influencing the overall survival of patients with liver cancer. In the multivariate analysis, the expression level of SLC17A2 was a significant independent prognostic biomarker for HCC (HR $=0.594, \mathrm{P}=0.01$, Table II).

Potential diagnostic value of SLC17A2 in HCC patients. We explored the diagnostic value of SLC17A2 in $\mathrm{HCC}$, and SLC17A2 expression was interrelated to HCC. In addition, the ROC curve was plotted and the area under the curve (AUC) was 0.708 . The $95 \% \mathrm{Cl}$ was between 0.656 and $0.760(\mathrm{P}<0.0001)$ (Fig. 4E), which revealed the diagnostic value of SLC17A2 in the HCC population.

Biological signaling pathways related to SLC17A2 according to GSEA. GSEA was used to predict the biological pathways related to SLC17A2. The first six enrichment pathways associated with SLC17A2 were fatty acid metabolism, valine leucine and isoleucine degradation, glycine serine and threonine 
metabolism, histidine metabolism, butanoate metabolism and peroxisome (Fig. 5A), suggesting that SLC17A2 is involved in many biological processes of HCC.

Gene Ontology (GO) and Kyoto Encyclopedia of Genes and Genomes (KEGG) functional enrichment analyses. First, the cBioPortal and UALCAN databases were screened, and 1142 genes (those with a Spearman's correlation coefficient $<0.3$ or $>0.3$ were removed) and 288 genes (those with a Pearson's correlation coefficient $<0.3$ or $>0.3$ were removed) that were coexpressed with SLC17A2 were obtained, respectively, and intersected with a Venn diagram (http://bioinformatics.psb.ugent.be/webtools/Venn/); ultimately, 158 coexpressed genes were obtained (Fig. 4F).

GO and KEGG analyses of the 158 coexpressed genes were performed with the "ggplot2" and "clusterProfiler" packages of R programming. GO analysis indicated that the 158 genes are mainly involved in fatty acid and cellular amino acid metabolism and carboxylic acid and organic acid catabolism (biological processes) microbodies and peroxisomes (cellular components) and coenzyme binding and oxidoreductase activity (molecular functions) (Fig. 5B). The top 30 pathways analyzed are shown in Fig. 6. The intersecting genes mainly participate in the peroxisome, complement and coagulation cascade, carbohydrate, fatty acid and amino acid metabolism, cytochrome P450 and chemical carcinogenesis pathways.

Construction of the PPI network and selection of hub genes. After identifying the disease-related genes, the STRING database was used to acquire the PPI network. Then, the network we obtained was further processed through Cytoscape, and the unconnected nodes were removed (Fig. 7A-B). In addition, we used the cytoHubba plug-in of Cytoscape to screen the top 10 genes through the Closeness algorithm, and the genes are shown in Fig. 7C. The top 10 hub genes are as follows: CAT, APOB, CYP3A4, AOX1, EHHADH, MAPK14, HSD17B4, PLG, NR1H4, CYP2E1.

Overall survival analysis according to the top three hub genes. We used the GEPIA database to perform overall survival analysis. The analysis of the CAT, APOB and CYP3A4 suggested a significant correlation between the survival time and CAT $(P<0.05)$ and CYP3A4 $(P<0.05)$ expression (Fig. 7D-F).

SLC17A2 expression is related to immune cells in the hepatocellular cancer microenvironment. Tumorinfiltrating immune cells (TIICs) are a part of the complex microenvironment and are related to the development of tumors[13]. We used the TIMER database to estimate SLC17A2 expression and the enrichment of TIICs, including B lymphocytes, CD $4+T$ cells, CD8 + T cells, regulatory $T$ cells (Treg), dendritic cells, neutrophils and macrophages. We found that SLC17A2 expression was positively associated with the infiltration levels of CD4 $+T$ cells $(R=0.153, p=4.38 e-3)$, naive CD8 + T cells $(R=$ $0.271, p=3.1 e-7)$, and naive $B$ cells $(R=0.128, p=0.017)$ and negatively correlated with the levels of Treg cells $(R=-0.171, p=0.001)$ in HCC (Fig. 8). These results indicate that SLC17A2 plays a significant role in the immune infiltration of HCC.

Correlations between SLC17A2 and immune cell markers. Finally, to deepen our understanding of immunity, in addition to analyzing common types of immune cells, we used the GEPIA database to 
further explore the correlations between SLC17A2 and markers of immunocyte to identify subtypes of immune cells. We analyzed both innate immune cells such as macrophages, neutrophils, NK cells, and dendritic cells, and various functional adaptive immune cells, such as B cells, CD $8+T$ cells, Th1 cells, Th2 cells, Th17 cells and Tregs, in HCC (Table III). The results suggested that SLC17A2 expression is closely associated with most makers of various immune cell types in HCC.

Excitingly, we found that SLC17A2 expression was implicated in the levels of some gene marker sets of CD8 + T cells, Th1 cells, Th2 cells, Th17 cells, Tregs, B cells, neutrophils, macrophages and NK cells. More specifically, we found that ABT1, ZNF22, and ZEB1 of CD8 + T cells, DPP4 of Th1 cells, MICAL3 and BACH2 of B cells, SGMS1 of macrophages, CYP4F3, HIST1H2BC, and KIAA0329 of neutrophils, IL17A and STAT3 of Th17 cells, and CDC5L, ALDH1B1, and ARL6IP2 of NK cells are significant positively correlated with SLC17A2 expression in HCC $(P<0.05)$. We also discovered the significant negative correlations between marker genes of Th2 and Treg cells and SLC17A2 expression $(P<0.05)$, in accordance with the results from the TIMER database (Fig. 9). In conclusion, SLC17A2 may affect the development of $\mathrm{HCC}$ by regulating the infiltration of immune cells with different phenotypes and may explain the effect of SLC17A2 on the prognosis of HCC.

\section{Discussion}

Although there are many studies on liver cancer, due to the occult onset of liver cancer, the postoperative metastasis rate and drug resistance rate remain high. Therefore, it is urgent to identify a new prognostic marker for HCC. Our study combined basic experiments with bioinformatics methods to show that SLC17A2 may be a valuable prognostic marker in HCC. This is the first comprehensive analysis of SLC17A2 expression in HCC, and it is the first study to prove that SLC17A2 protein expression is significantly downregulated in HCC by immunohistochemistry. In addition, SLC17A2 expression was closely correlated with prognosis, the overall survival time of patients with low SLC17A2 expression was shorter than that of patients with high SLC17A2 expression, and multivariate Cox analysis indicated that SLC17A2 is an independent prognostic factor of HCC, besides, the ROC curve demonstrated that SLC17A2 has high diagnostic value. These results suggest that SLC17A2 acts as a tumor suppressor gene in the development and progression of liver cancer.

Next, we explored the metabolic pathway related to SLC17A2 in liver cancer. GSEA showed that SLC17A2 expression is positively associated with the peroxisome metabolism, fatty acid metabolism and amino acid metabolism pathways; that is, the abovementioned metabolic pathways are inhibited in liver cancer tissue. Some studies have shown that peroxisomes are very important to the metabolism of hepatoma cells; their oxidation is regulated by the classic peroxisome proliferator induction pathway[14], and the dysfunction of peroxisome metabolism in liver cancer patients' tissues will lead to an increase in active oxygen levels involved in various cancer signaling pathways. Moreover, the high oxidative stress of cells will affect cell sugar metabolism and increase autophagy $[15,16]$. Autophagy was initially believed to inhibit tumors[17], but interestingly, autophagy in the liver is necessary for tumor development. The various metabolic fuels produced by autophagy provide metabolic plasticity for the tumor and enable 
tumor cells to thrive in the immune microenvironment[18]. Autophagy can degrade carbohydrates, promote glycolysis, promote fatty acid metabolism, and target protein conversion into amino acids[19]. Given the increasing metabolic demand of tumor cells, metabolic pathways are especially important for the proliferation of cancer cells, among which lipid metabolism has been proven to be tightly related to $\mathrm{HCC}[20]$. The liver is a significant place for amino acid metabolism; generally speaking, these amino acids participate in tumor cell metabolism and nucleic acid and lipid synthesis[21]. However, an investigation of free amino acids in plasma by some researchers showed that the histidine content in liver cancer patients was decreased, indicating that amino acid metabolism is disordered in liver cancer[22]. Serine is also relatively special amino acids. It is closely related to the formation of nucleic acid precursors in cancer cells[23,24]. In vitro experiments have shown that an increase in its concentration can reduce the proliferation of cancer cells[25], and in the future, we can explore a new anticancer method by changing the metabolism of amino acids and peroxidase.

Tumor immunotherapy has rapidly evolved in recent years, so it is of great significance to explore prognostic and diagnostic biomarkers and targeted therapies. Numerous researches have shown that TIICs are related to the development and progression of HCC, and immune cell function is closely associated with metabolism[26]. Our study is the first to reveal that SLC17A2 expression is positively related to the infiltration levels of $C D 4+T$ cells, naive $C D 8+T$ cells and naive $B$ cells and negatively correlated with the levels of Treg cells, suggesting that SLC17A2 is closely related to the recruitment and regulation of immunocyte in HCC. In the tumor microenvironment of HCC, the antitumor function of immune cells is impaired due to restricted metabolism. However, Tregs are a special subset of CD4 + T cells that are different from conventional $\mathrm{T}$ cells and play an immunosuppressive role in the tumor microenvironment[27]. In addition, Bettina Langhans et al. indicated that the levels of IL-10 and IL-35 secreted by Tregs in the blood of patients with HCC were remarkably higher than those of normal cells[17], and Treg infiltration in HCC was associated with a poor prognosis[25]. In addition, in the early stage of tumorigenesis, naive CD8 + T cells are dysfunctional, showing similar phenotype, function and transcription characteristics to dysfunctional T cells in advanced tumors, indicating that $\mathrm{T}$ cell dysfunction occurs in the early stage of $\mathrm{HCC}[28]$. In addition, it has been reported that the selective loss of CD $4+T$ cells in the tumor tissue of HCC patients accelerates the development of liver cancer[29]. In addition, as the main component of the tumor immune microenvironment, a high density of tumorinfiltrating naive $B$ cells is associated with a high survival rate[30]. In conclusion, our study indicates that SLC17A2 may improve the prognosis of HCC by recruiting immune cells and provides a detailed characterization of the association between SLC17A2 and immune markers in HCC patients. The exact role of SLC17A2 in the tumor immune microenvironment and whether it is a key factor in immunotherapy need further exploration.

The top 3 hub genes, CAT, APOB and CYP3A4, are closely related to the occurrence and development of liver cancer. $\mathrm{K}$ Suto et al. indicated a decrease in catalase (CAT) activity in HCC that was caused by the inhibition of CAT gene transcription[31]. Gena Lee et al. reported that decrease of APOB expression notably increased the multiplication of hepatoma cells, suggesting that APOB is a tumor suppressor gene[32]. Ryo Ashida et al. showed that carcinogenesis easily occurs in HCC patients with downregulated 
CYP3A4 expression, which was associated with high exposure to precarcinogens caused by low CYP3A4 expression.[33] In addition, the survival analysis suggested that CAT and CYP3A4 were significant for prognosis. Taken together, these results show that the tumor-regulated signaling pathways have multiple genes that work together; therefore, SLC17A2 may cooperate with CAT, APOB and CYP3A4 to regulate tumor metabolism pathways, but these results were predicted from bioinformatics analysis and thus need to be verified experimentally.

In our study, we first reported that low SLC17A2 expression is closely related to the poor prognosis of liver cancer. Moreover, the diagnostic value and independent prognostic potential of SLC17A2 were confirmed. Our study provides a comprehensive and reliable theoretical basis for subsequent studies of the relationship between SLC17A2 and liver cancer

However, our study has some limitations. First, some patients whose data were obtained from the TCGA and GEO databases were still in follow-up; therefore, the data were incomplete. Our goal is to collect samples for verification in the future. Second, this study did not accurately evaluate the biological function and detailed regulatory mechanism of SLC17A2 in liver cancer, and these deficiencies will be improved and studied in future experiments.

\section{Conclusions}

SLC17A2 expression is downregulated in HCC, strongly correlates with HCC prognosis, and has independent diagnostic value.

\section{Declarations}

\section{Acknowledgements}

Not applicable.

\section{Authors' contributions}

ZJ and ZW were the designer and mastermind of this study. ZW analyzed and processed the data. ZW draw up the manuscript. ZJ and XC modified and polished the manuscript. All authors read and approved this manuscript.

\section{Funding}

No funding.

\section{Availability of data and materials}

The datasets acquired and analyzed during the current study from the TCGA database (https://mirrors.tuna.tsinghua.edu.cn/CRAN/) and GSE45267, GSE14520 and GSE54236 of GEO 
database (https://www. ncbi.nlm.nih.gov/gds/), and this data are available from the corresponding authors upon request.

\section{Ethics approval and consent to participate}

All the samples were obtained the informed consent of the patients and their families, and the procedures were examined by the Ethics Committee of the First Affiliated Hospital of Chongqing Medical University.

\section{Consent for publication}

Not applicable.

\section{Competing interests}

The authors declare that they have no competing interests.

\section{References}

1. An L, Zeng HM, Zheng RS, Zhang SW, Sun KX, Zou XN, Chen R, Wang SM, Gu XY, Wei WW, He J. [Liver cancer epidemiology in China, 2015]. Zhonghua Zhong Liu Za Zhi. 2019;41:721-7.

2. Villanueva A. Hepatocellular Carcinoma. N Engl J Med. 2019;380:1450-62.

3. Spolverato G, Bagante F, Weiss M, Alexandrescu S, Marques HP, Aldrighetti L, Maithel SK, Pulitano C, Bauer TW, Shen F, et al: Comparative performances of the 7th and the 8th editions of the American Joint Committee on Cancer staging systems for intrahepatic cholangiocarcinoma. J Surg Oncol 2017, 115:696-703.

4. Kang SH, Hwang S, Lee YJ, Kim KH, Ahn CS, Moon DB, Ha TY, Song GW, Jung DH, Lee SG: Prognostic comparison of the 7th and 8th editions of the American Joint Committee on Cancer staging system for intrahepatic cholangiocarcinoma. J Hepatobiliary Pancreat Sci 2018, 25:240-248.

5. Zhang Y, Zhang Y, Sun K, Meng Z, Chen L. The SLC transporter in nutrient and metabolic sensing, regulation, and drug development. J Mol Cell Biol. 2019;11:1-13.

6. Bissa B, Beedle AM, Govindarajan R. Lysosomal solute carrier transporters gain momentum in research. Clin Pharmacol Ther. 2016;100:431-6.

7. Song W, Li D, Tao L, Luo Q, Chen L. Solute carrier transporters: the metabolic gatekeepers of immune cells. Acta Pharm Sin B. 2020;10:61-78.

8. Ren A, Sun S, Li S, Chen T, Shu Y, Du M, Zhu L. Genetic variants in SLC22A3 contribute to the susceptibility to colorectal cancer. Int J Cancer. 2019;145:154-63.

9. El Ansari R, Craze ML, Diez-Rodriguez M, Nolan CC, Ellis IO, Rakha EA, Green AR. The multifunctional solute carrier 3A2 (SLC3A2) confers a poor prognosis in the highly proliferative breast cancer subtypes. Br J Cancer. 2018;118:1115-22.

10. Liu R, Hong R, Wang Y, Gong Y, Yeerken D, Yang D, Li J, Fan J, Chen J, Zhang W, Zhan Q. Defect of SLC38A3 promotes epithelial-mesenchymal transition and predicts poor prognosis in esophageal 
squamous cell carcinoma. Chin J Cancer Res. 2020;32:547-63.

11. Ruiz-Deya G, Matta J, Encarnacion-Medina J, Ortiz-Sanchez C, Dutil J, Putney R, Berglund A, Dhillon J, Kim Y, Park JY. Differential DNA Methylation in Prostate Tumors from Puerto Rican Men. Int J Mol Sci 2021, 22.

12. Bien SA, Pankow JS, Haessler J, Lu Y, Pankratz N, Rohde RR, Tamuno A, Carlson CS, Schumacher FR, Buzkova $P$, et al. Transethnic insight into the genetics of glycaemic traits: fine-mapping results from the Population Architecture using Genomics and Epidemiology (PAGE) consortium. Diabetologia. 2017;60:2384-98.

13. Domingues P, Gonzalez-Tablas M, Otero A, Pascual D, Miranda D, Ruiz L, Sousa P, Ciudad J, Goncalves JM, Lopes MC, et al. Tumor infiltrating immune cells in gliomas and meningiomas. Brain Behav Immun. 2016;53:1-15.

14. Reddy JK, Hashimoto T. Peroxisomal beta-oxidation and peroxisome proliferator-activated receptor alpha: an adaptive metabolic system. Annu Rev Nutr. 2001;21:193-230.

15. Cai M, Sun X, Wang W, Lian Z, Wu P, Han S, Chen H, Zhang P. Disruption of peroxisome function leads to metabolic stress, mTOR inhibition, and lethality in liver cancer cells. Cancer Lett. 2018;421:82-93.

16. Chen XF, Tian MX, Sun RQ, Zhang ML, Zhou LS, Jin L, Chen LL, Zhou WJ, Duan KL, Chen YJ, et al: SIRT5 inhibits peroxisomal ACOX1 to prevent oxidative damage and is downregulated in liver cancer. EMBO Rep 2018, 19.

17. Langhans B, Nischalke HD, Kramer B, Dold L, Lutz P, Mohr R, Vogt A, Toma M, Eis-Hubinger AM, Nattermann J, et al. Role of regulatory T cells and checkpoint inhibition in hepatocellular carcinoma. Cancer Immunol Immunother. 2019;68:2055-66.

18. Kimmelman AC, White E. Autophagy and Tumor Metabolism. Cell Metab. 2017;25:1037-43.

19. Rabinowitz JD, White E. Autophagy and metabolism. Science. 2010;330:1344-8.

20. Pope ED 3rd, Kimbrough EO, Vemireddy LP, Surapaneni PK, Copland JA 3rd, Mody K. Aberrant lipid metabolism as a therapeutic target in liver cancer. Expert Opin Ther Targets. 2019;23:473-83.

21. Lee DY, Kim EH. Therapeutic Effects of Amino Acids in Liver Diseases: Current Studies and Future Perspectives. J Cancer Prev. 2019;24:72-8.

22. Ye AL, Tang ZY, Liu H, Zhao QR, Zhu WN. [Alteration of plasma amino acid content in primary liver cancer patients]. Zhonghua Zhong Liu Za Zhi. 1987;9:190-2.

23. Snell K, Weber G. Enzymic imbalance in serine metabolism in rat hepatomas. Biochem J. 1986;233:617-20.

24. Snell K. Enzymes of serine metabolism in normal, developing and neoplastic rat tissues. Adv Enzyme Regul. 1984;22:325-400.

25. Li F, Guo Z, Lizee G, Yu H, Wang H, Si T. Clinical prognostic value of CD4 + CD25 + FOXP3 + regulatory $\mathrm{T}$ cells in peripheral blood of Barcelona Clinic Liver Cancer (BCLC) stage B hepatocellular carcinoma patients. Clin Chem Lab Med. 2014;52:1357-65. 
26. Zhang Q, Lou Y, Bai XL, Liang TB. Immunometabolism: A novel perspective of liver cancer microenvironment and its influence on tumor progression. World J Gastroenterol. 2018;24:3500-12.

27. Pacella I, Piconese S. Immunometabolic Checkpoints of Treg Dynamics: Adaptation to Microenvironmental Opportunities and Challenges. Front Immunol. 2019;10:1889.

28. Schietinger A, Philip M, Krisnawan VE, Chiu EY, Delrow JJ, Basom RS, Lauer P, Brockstedt DG, Knoblaugh SE, Hammerling GJ, et al. Tumor-Specific T Cell Dysfunction Is a Dynamic Antigen-Driven Differentiation Program Initiated Early during Tumorigenesis. Immunity. 2016;45:389-401.

29. Ma C, Kesarwala AH, Eggert T, Medina-Echeverz J, Kleiner DE, Jin P, Stroncek DF, Terabe M, Kapoor V, ElGindi $\mathrm{M}$, et al. NAFLD causes selective CD4(+) T lymphocyte loss and promotes hepatocarcinogenesis. Nature. 2016;531:253-7.

30. Zhang Z, Ma L, Goswami S, Ma J, Zheng B, Duan M, Liu L, Zhang L, Shi J, Dong L, et al. Landscape of infiltrating $B$ cells and their clinical significance in human hepatocellular carcinoma. Oncoimmunology. 2019;8:e1571388.

31. Sato K, Ito K, Kohara H, Yamaguchi Y, Adachi K, Endo H. Negative regulation of catalase gene expression in hepatoma cells. Mol Cell Biol. 1992;12:2525-33.

32. Lee G, Jeong YS, Kim DW, Kwak MJ, Koh J, Joo EW, Lee JS, Kah S, Sim YE, Yim SY. Clinical significance of APOB inactivation in hepatocellular carcinoma. Exp Mol Med. 2018;50:1-12.

33. Ashida R, Okamura Y, Ohshima K, Kakuda Y, Uesaka K, Sugiura T, Ito T, Yamamoto Y, Sugino T, Urakami K, et al. CYP3A4 Gene Is a Novel Biomarker for Predicting a Poor Prognosis in Hepatocellular Carcinoma. Cancer Genomics Proteomics. 2017;14:445-53.

\section{Tables}

Table I. Statistical results of immunohistochemistry for SLC17A2 protein expression in HCC tissues and normal liver tissues.

\begin{tabular}{|lllll|}
\hline \multicolumn{2}{|c|}{ Tissue } & \multirow{2}{*}{ X2 P value } \\
& Normal(n=28) & Cancer(n=32) & \\
\hline Immunohistochemical grade & & & & \\
High expression of SLC17A2 & $22(78.6 \%)$ & $3(9.4 \%)$ & $29.418<0.0001$ \\
Low expression of SLC17A2 & $6(21.4 \%)$ & $29(90.6 \%)$ & & \\
\hline
\end{tabular}

Table II: SLC17A2 expression and other clinicopathological parameters of HCC analyzed by multivariate and univariate cox regression analyses 


\begin{tabular}{|c|c|c|c|c|c|c|}
\hline \multirow[t]{2}{*}{ Characteristic } & \multicolumn{3}{|c|}{ Univariate analysis } & \multicolumn{3}{|c|}{ Multivariate analysis } \\
\hline & $\mathrm{HR}$ & $95 \% \mathrm{Cl}$ & P-value & $\mathrm{HR}$ & $95 \% \mathrm{Cl}$ & P-value \\
\hline \multicolumn{7}{|l|}{ Age $\Downarrow$ years $\rrbracket$} \\
\hline$<60$ & 1 & - & - & 1 & - & - \\
\hline$\geq 60$ & 1.313 & $0.906-1.902$ & 0.15 & 1.312 & $0.895-1.924$ & 0.164 \\
\hline \multicolumn{7}{|l|}{ Gender } \\
\hline Male & 1 & - & - & 1 & - & - \\
\hline Female & 1.297 & $0.891-1.888$ & 0.175 & 1.128 & $0.760-1.674$ & 0.549 \\
\hline \multicolumn{7}{|l|}{ T stage } \\
\hline 1 & 1 & - & - & 1 & - & - \\
\hline 2 & 1.339 & $0.817-2.194$ & 0.247 & 0.002 & $<0.0001$ & 0.796 \\
\hline 3 & 2.545 & $1.652-3.921$ & $<0.0001$ & 4.324 & $0.256-73.173$ & 0.310 \\
\hline 4 & 4.920 & $2.192-11.047$ & $<0.0001$ & 7.119 & $0.354-143.142$ & 0.200 \\
\hline \multicolumn{7}{|l|}{ N stage } \\
\hline 0 & 1 & - & - & 1 & - & - \\
\hline 1 & 1.944 & $0.479-7.899$ & 0.353 & 3.773 & $0.503-28.269$ & 0.196 \\
\hline \multicolumn{7}{|l|}{ M stage } \\
\hline 0 & 1 & - & - & 1 & - & - \\
\hline 1 & 3.752 & $1.187-11.858$ & 0.024 & 429.727 & $<0.0001$ & 0.968 \\
\hline \multicolumn{7}{|l|}{ TNM stage } \\
\hline ૫ & 1 & - & - & 1 & - & - \\
\hline ૫ & 1.383 & $0.842-2.270$ & 0.200 & 793.455 & $<0.0001$ & 0.789 \\
\hline ૫ & 2.659 & $1.743-4.056$ & $<0.0001$ & 0.587 & $0.033-10.297$ & 0.716 \\
\hline ૫ & 5.417 & $1.671-17.560$ & 0.005 & 0.001 & $<0.0001$ & 0.965 \\
\hline \multicolumn{7}{|c|}{ SLC17A2 expression } \\
\hline Low & 1 & - & - & 1 & - & - \\
\hline High & 0.590 & $0.398-0.874$ & 0.009 & 0.594 & $0.399-0.885$ & 0.010 \\
\hline
\end{tabular}

HR, hazard ratio; Cl: confidence interval. 
Table III: The correlation analysis between the expression of immune cells markers and SLC17A2 by Pearson test. 
Description Gene maker HCC

\begin{tabular}{|c|c|c|c|c|c|}
\hline & & Tumor & & Normal & \\
\hline & & $\mathbf{R}$ & $\mathbf{P}$ & $\mathbf{R}$ & $\mathbf{P}$ \\
\hline \multirow[t]{4}{*}{ CD8+ T cell } & SLC16A7 & 0.085 & 0.064 & 0.32 & $\star \star \star *$ \\
\hline & $\mathrm{ABT} 1$ & 0.25 & $\star \star \star$ & 0.12 & 0.13 \\
\hline & ZNF22 & 0.2 & $\star \star \star *$ & 0.3 & $\star \star \star$ \\
\hline & ZEB1 & 0.18 & $\star \star \star$ & 0.0013 & 0.99 \\
\hline \multirow[t]{2}{*}{ Th1 } & CD38 & -0.027 & 0.56 & 0.17 & * \\
\hline & DPP4 & 0.22 & $\star * *$ & 0.056 & 0.48 \\
\hline \multirow[t]{5}{*}{ Th2 } & $\mathrm{PMCH}$ & -0.13 & ** & -0.2 & $\star *$ \\
\hline & LAIR2 & -0.1 & * & -0.17 & * \\
\hline & HELLS & -0.15 & $\star \star \star \star$ & -0.056 & 0.48 \\
\hline & SNRPD1 & -0.18 & $\star \star \star \star ~$ & 0.074 & 0.35 \\
\hline & BIRC5 & -0.2 & $\star \star \star$ & 0.027 & 0.74 \\
\hline \multirow[t]{2}{*}{ Treg } & TGFB1 & -0.17 & $\star \star * \star$ & -0.2 & * \\
\hline & FOXP3 & 0.077 & 0.09 & -0.077 & 0.33 \\
\hline \multirow[t]{3}{*}{ B cell } & GLDC & 0.11 & 0.17 & 0.071 & 0.12 \\
\hline & MICAL3 & 0.22 & $\star * *$ & 0.099 & 0.21 \\
\hline & $\mathrm{BACH} 2$ & 0.19 & $\star \star \star \star$ & 0.43 & $\star \star \star$ \\
\hline \multirow[t]{2}{*}{ Dendritic cell } & HSD11B1 & 0.11 & * & 0.11 & 0.17 \\
\hline & PPFIBP2 & 0.11 & * & 0.005 & 0.95 \\
\hline \multirow[t]{3}{*}{ Macrophages } & CHI3L1 & 0.081 & 0.076 & 0.3 & $* \star *$ \\
\hline & FDX1 & 0.1 & * & 0.055 & 0.49 \\
\hline & SGMS1 & 0.14 & ** & 0.035 & 0.66 \\
\hline \multirow[t]{3}{*}{ Neutrophils } & CYP4F3 & 0.15 & $\star \star \star *$ & 0.19 & * \\
\hline & HIST1H2BC & 0.21 & $\star * *$ & 0.33 & $\star \star \star *$ \\
\hline & KIAA0329 & 0.16 & $\star \star *$ & 0.15 & 0.066 \\
\hline
\end{tabular}




\begin{tabular}{|c|c|c|c|c|c|}
\hline Th17 cells & IL17A & 0.18 & $\star \star \star$ & 0.064 & 0.42 \\
\hline & STAT3 & 0.12 & $\star \star$ & 0.36 & $\star \star \star *$ \\
\hline \multirow[t]{4}{*}{ NK cell } & CDC5L & 0.37 & $\star \star \star$ & 0.15 & 0.061 \\
\hline & ALDH1B1 & 0.17 & $\star \star \star$ & 0.042 & 0.59 \\
\hline & ARL6IP2 & 0.19 & $\star \star \star$ & 0.41 & $\star \star \star$ \\
\hline & MAPRE3 & 0.09 & * & 0.21 & ** \\
\hline
\end{tabular}

HCC $\triangle H$ Hepatocellular carcinoma $\$ Tumor》correlation analysis of SLC17A2 and immune cells markers in

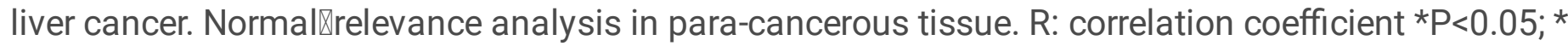
$* \mathrm{P}<0.01 ; * * * \mathrm{P}<0.001$

\section{Figures}

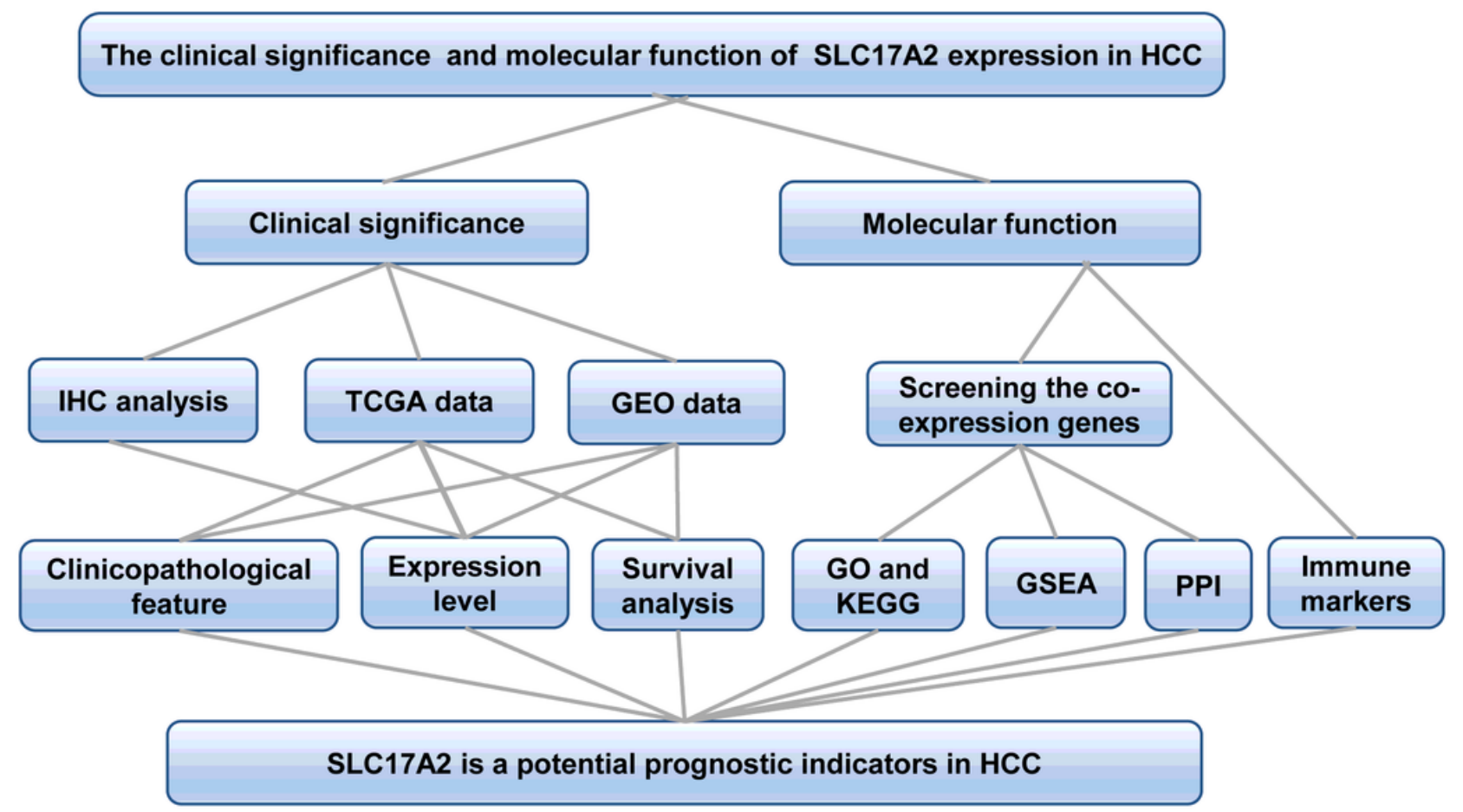

\section{Figure 1}

Flow chart of the research design 
A
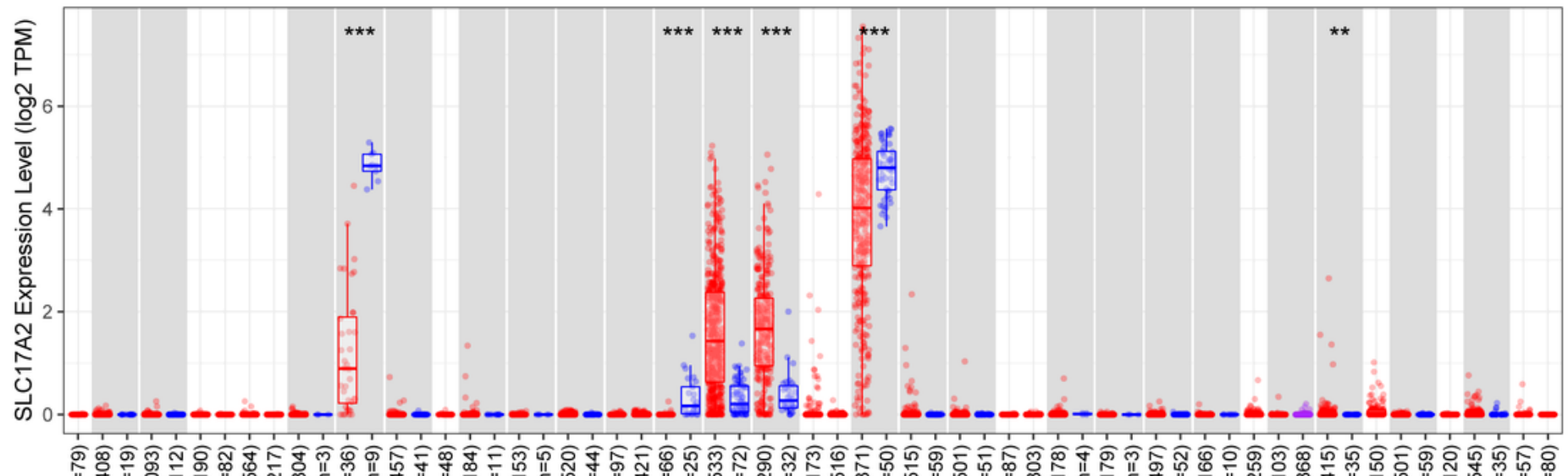

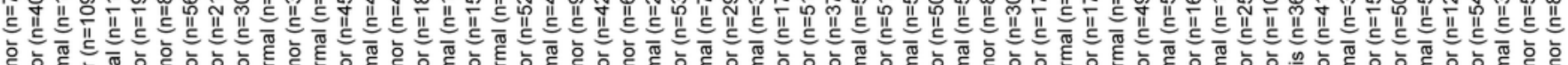

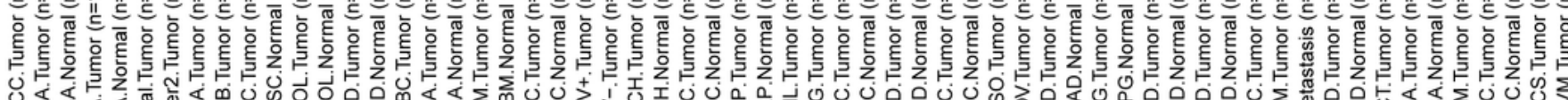

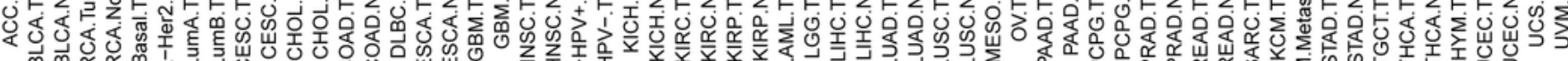

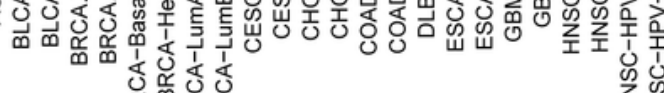

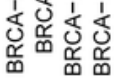

B

$\begin{array}{rrr}\text { Median Rank } & \text { p-Value } & \text { Gene } \\ 459.0 & 4.26 \mathrm{E}-7 & \text { SLC17A2 }\end{array}$

$$
\begin{array}{|l|l|l|l|l|l|l|}
\hline 1 & 2 & 3 & 4 & 5 & 6 & 7 \\
\hline
\end{array}
$$

The rank for a gene is the median rank for that gene across each of the analyses. The $p$-Value for a gene is its $p$-Value for the median-ranked analysis.

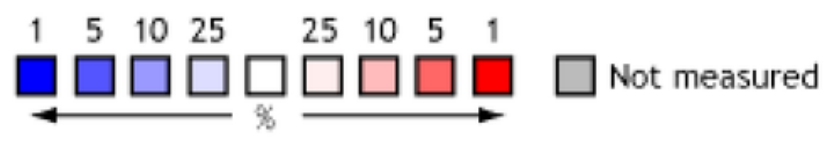

C

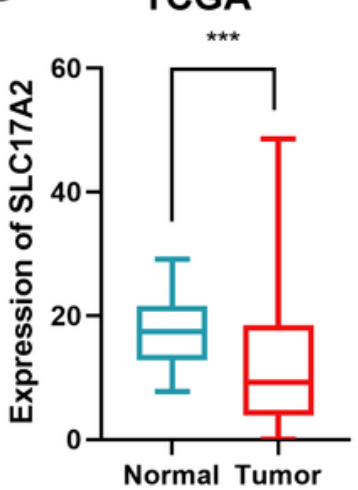

D

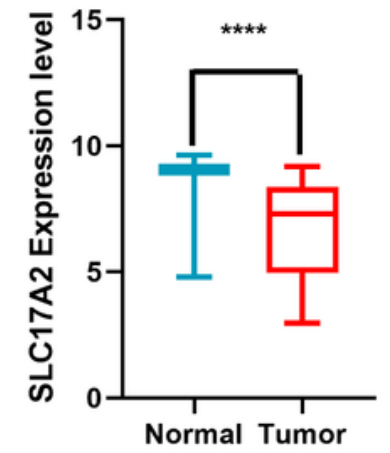

Legend

1. Hepatocellular Carcinoma vs. Normal 6 . Cirrhosis vs. Normal

Chen Liver, Mol Biol Cell, 2002

2. Cirrhosis vs. Normal

Wurmbach Liver, Hepatology, 2007

7. Hepatocellular Carcinoma vs. Normal

Mas Liver, Mol Med, 2008

3. Hepatocellular Carcinoma vs. Normal

Mas Liver, Mol Med, 2008

4. Hepatocellular Carcinoma vs. Normal

Roessler Liver, Cancer Res, 2010

5. Hepatocellular Carcinoma vs. Normal

Roessler Liver 2, Cancer Res, 2010

E

GSE14520

$\mathrm{F}$

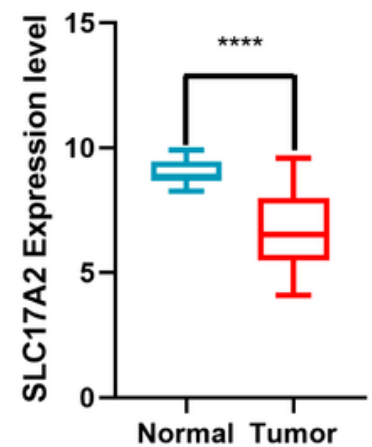

Wurmbach Liver, Hepatology, 2007

\section{Figure 2}

A. SLC17A2 is significantly expressed at low levels in HCC tumor tissues in the TCGA database, ${ }^{\star} * \star P<0.001,{ }^{\star *} P<0.01$. B. Studies on SLC17A2 expression in HCC are marked 1-7; blue represents the level of SLC17A2 expression, and as the expression increases, the color deepens. C-F. The expression of SLC17A2 in HCC tissue and normal tissue. C. Dataset from TCGA; D. Dataset from the GEO(GSE45267); 
E. Dataset from the GEO(GSE14520); F. Dataset from the GEO(GSE54236). Blue represents the adjacent normal tissue, and red represents the liver cancer tissue, ${ }^{\star *} P<0.01,{ }^{*} * * P<0.001, * \star \star \star P<0.0001$.

A

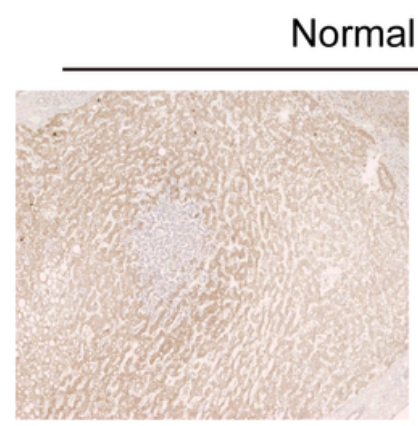

$\mathrm{B}$

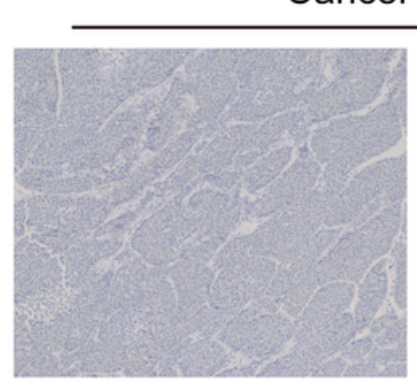

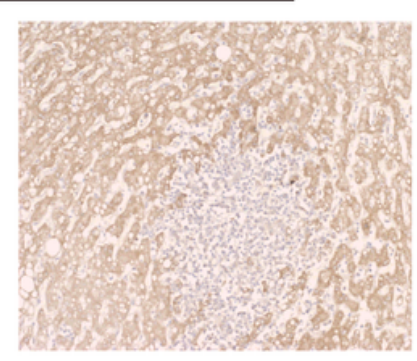

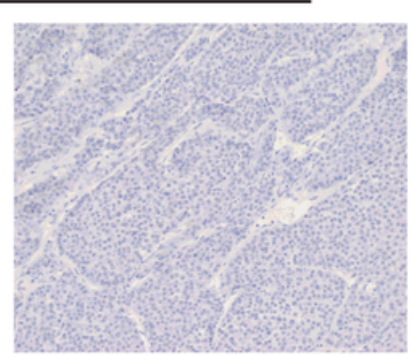

Figure 3

A-B. Normal and cancer images of SLC17A2 expression in liver tissues (100x and 400x) detected by IHC staining. 


\# Samples per Patient
Profiled in Mutations
$\begin{aligned} & \text { Profiled in Putative copy-number } \\ & \text { alterations from GISTIC }\end{aligned}$

B

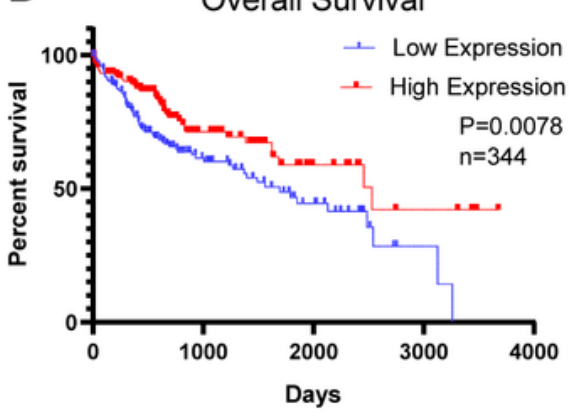

E

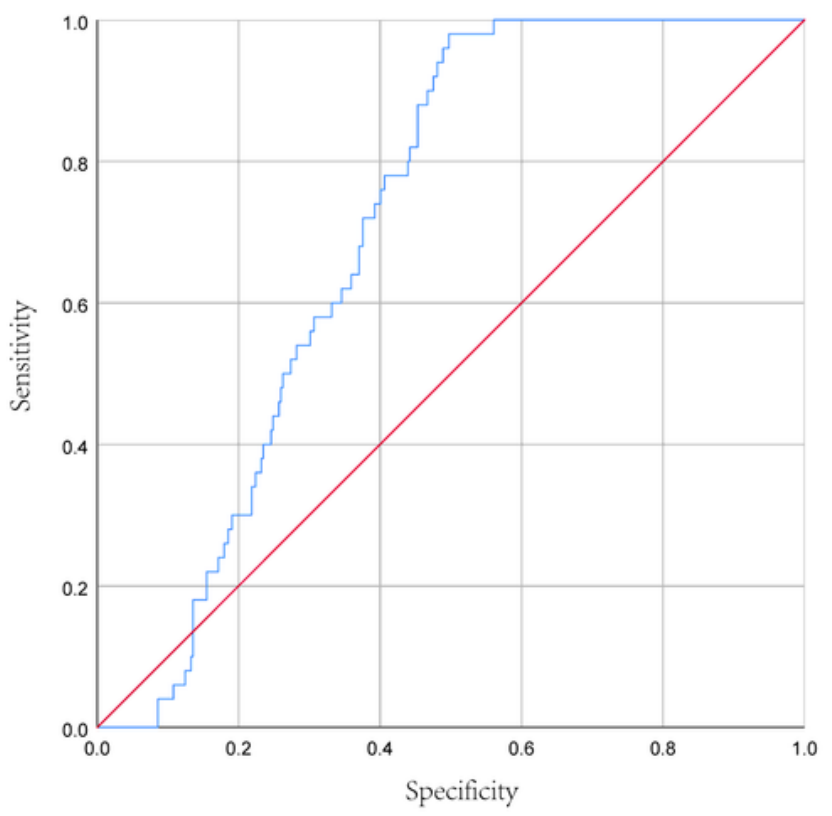

C

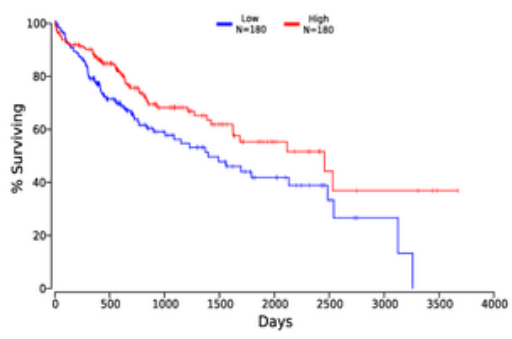

$\mathrm{F}$
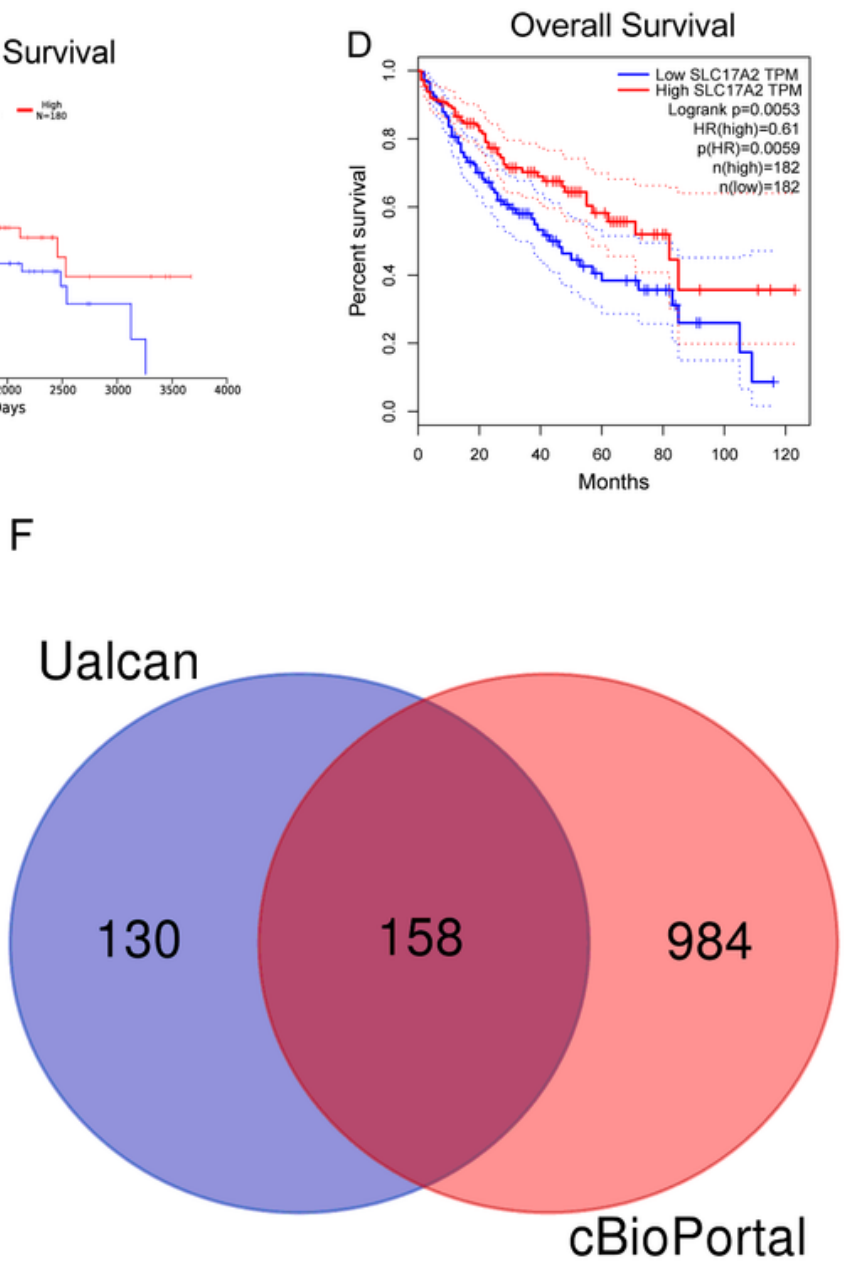

Figure 4

A. The OncoPrint schematic shows that SLC17A2 expression was altered in 4\% (16/400) of samples, including 14 cases of amplification; and 2 cases of a missense mutation. B-D. Kaplan-Meier analysis of SLC17A2 expression in patients with HCC, B: TCGA; C: GEPIA; D: OncoLnc. The red and blue lines indicate high expression and low expression, respectively. The abscissa and the ordinate represent the survival time and survival rate, respectively. E. ROC curve of SLC17A2 expression in HCC. The X and Y axes 
represent specificity and sensitivity, respectively. F. Venn diagram: the left panel was screened from UALCAN database, the right panel was screened from the cBioPortal database, and the dark red region in the middle panel represents the genes that are coexpressed with SLC17A2.

A

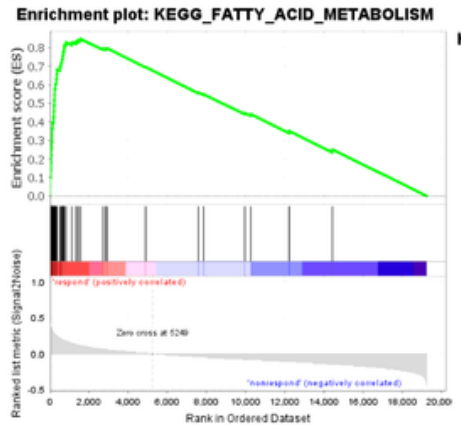

Enrichment plot:

Enrichment plot: $\quad$ Enrichment plot:

KEGG_VALINE_LEUCINE_AND_ISOLEUCINE_DEGRADA KEGG_GLYCINE_SERINE_AND_THREONINE_METABOLI
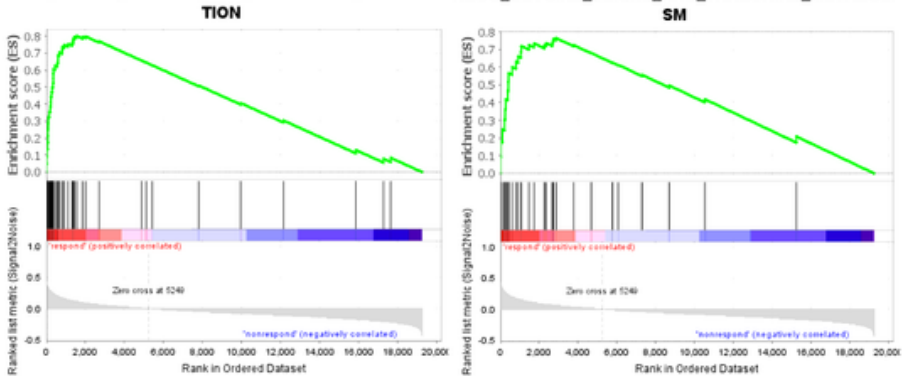

- Enichmentereste - Hits Ranking metic scores

Enrichment plot: KEGG_HISTIDINE_METABOLISM

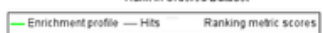

Enrichment plot: KEGG_BUTANOATE_METABOLISM

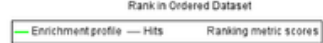

Enrichment plot: KEGG_PEROXISOME
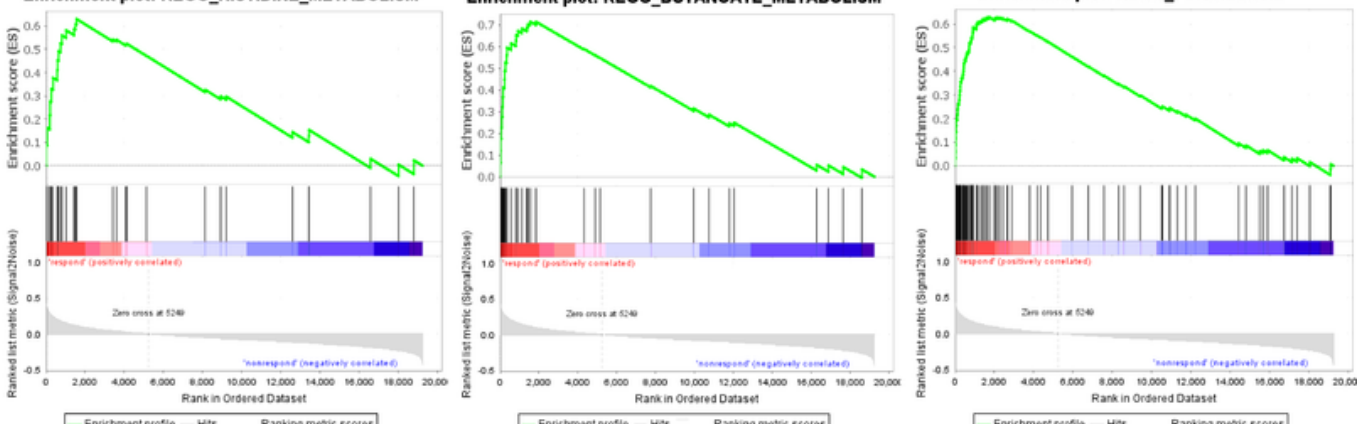

B

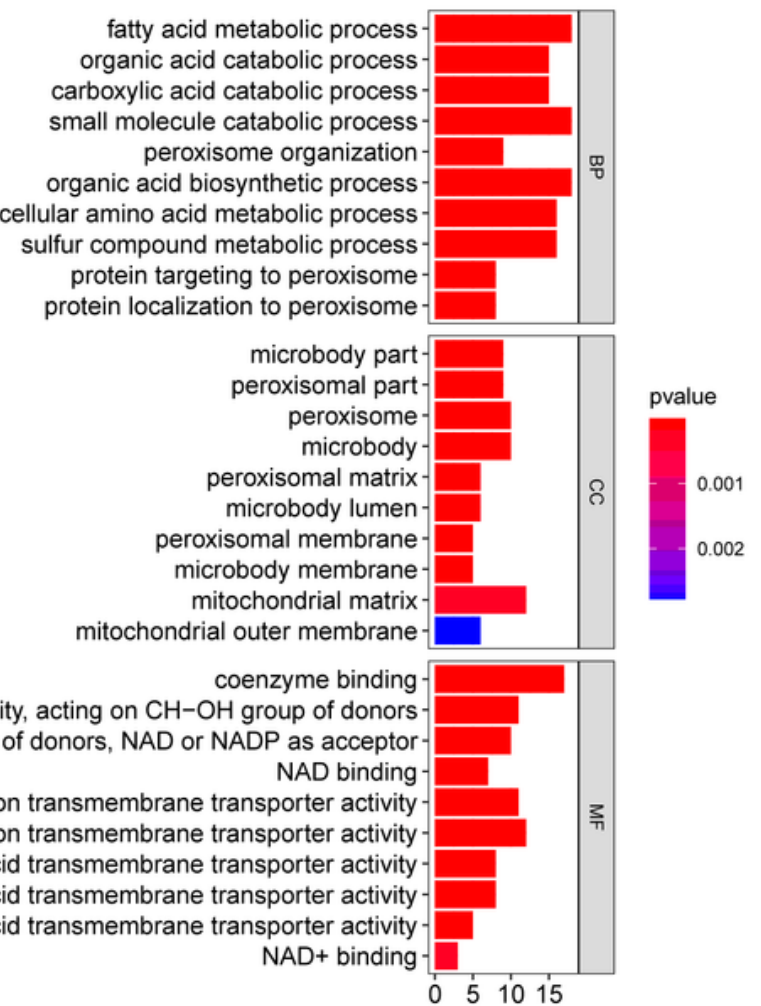

Figure 5

A. The top six enrichment pathways related to SLC17A2 are listed, and we found that SLC17A2 was related mainly to metabolism pathways. B. GO enrichment analysis: BP, CC, and MF represent biological 
process, cellular component, and molecular function, respectively. The length of the rectangle represents gene counts and the color represents the $P$ value.

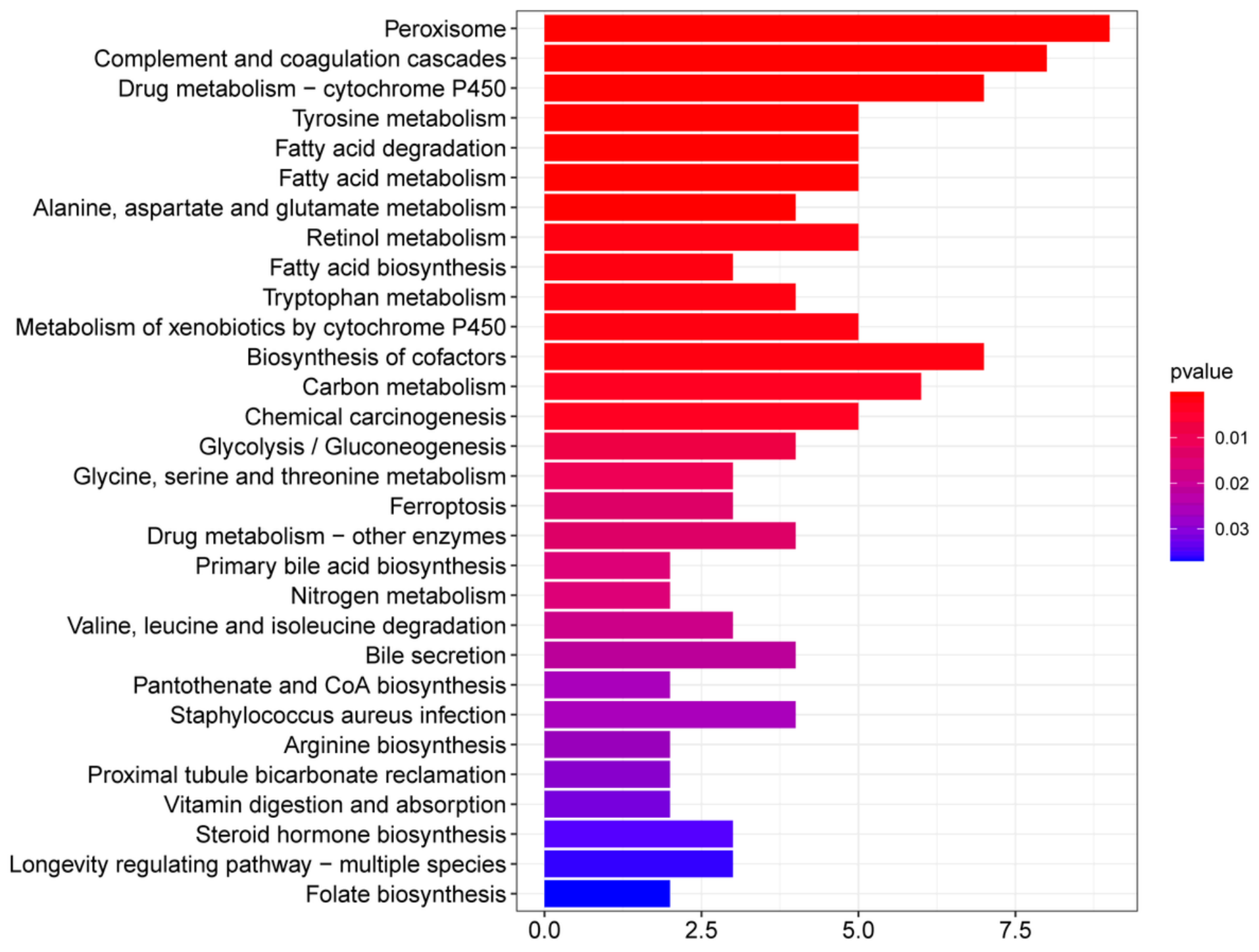

\section{Figure 6}

KEGG enrichment analysis: the length of the rectangle represents gene counts, and the color represents the $P$ value. 
A

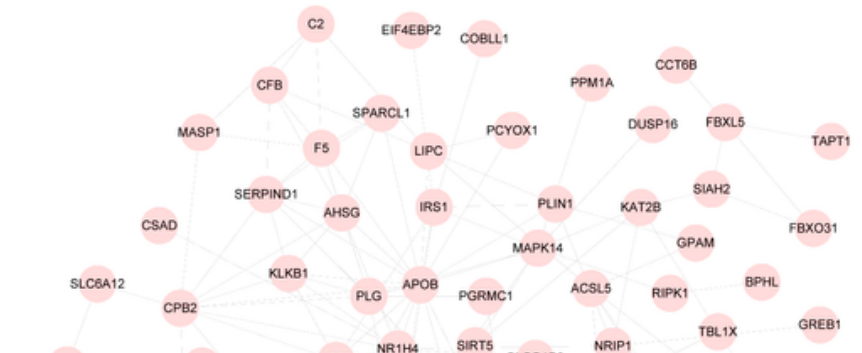

$$
\begin{aligned}
& \text { SLCBA13 }
\end{aligned}
$$

B

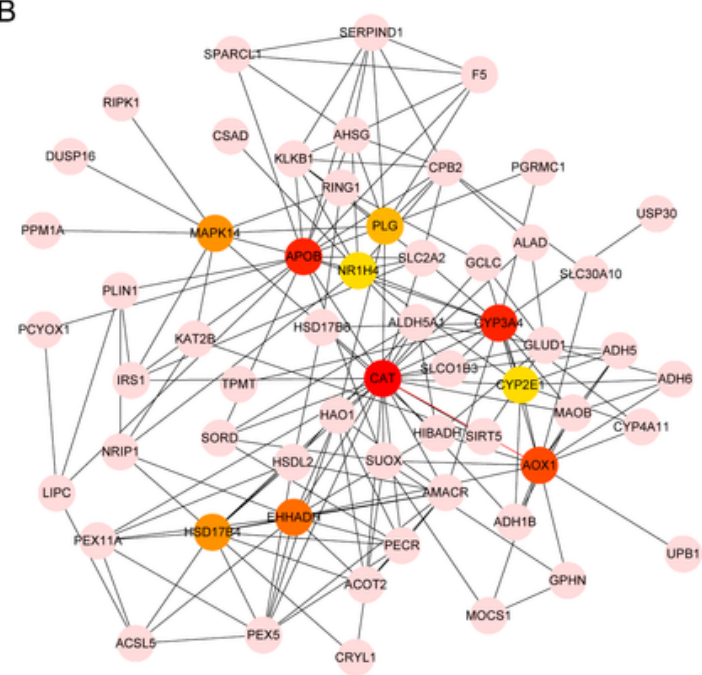

C

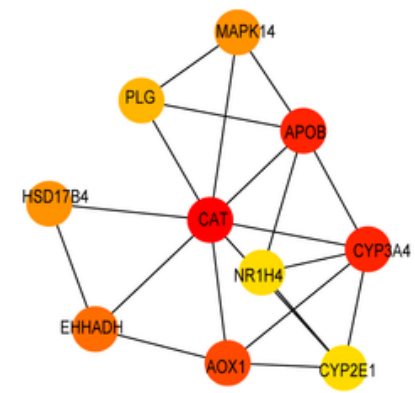

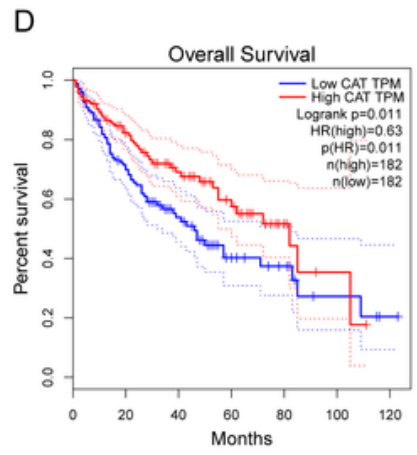

E

Overall Survival

F
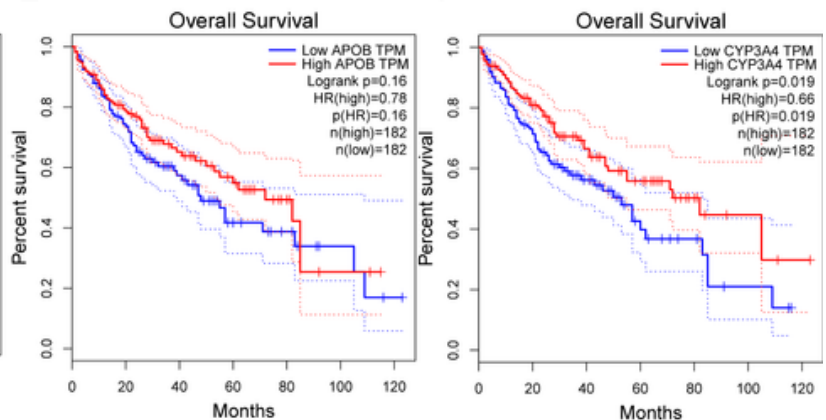

Figure 7

A. Protein-protein interaction (PPI) network of genes that are coexpressed with SLC17A2. B. The top ten hub genes are marked. C. The top ten hub genes screened from the PPI network. D-F. The Kaplan-Meier analysis according to each of the top three types of hub genes; red and blue lines represent high expression and low expression, respectively. D. CAT gene. E. APOB gene. F. CYP3A4 gene. 
A
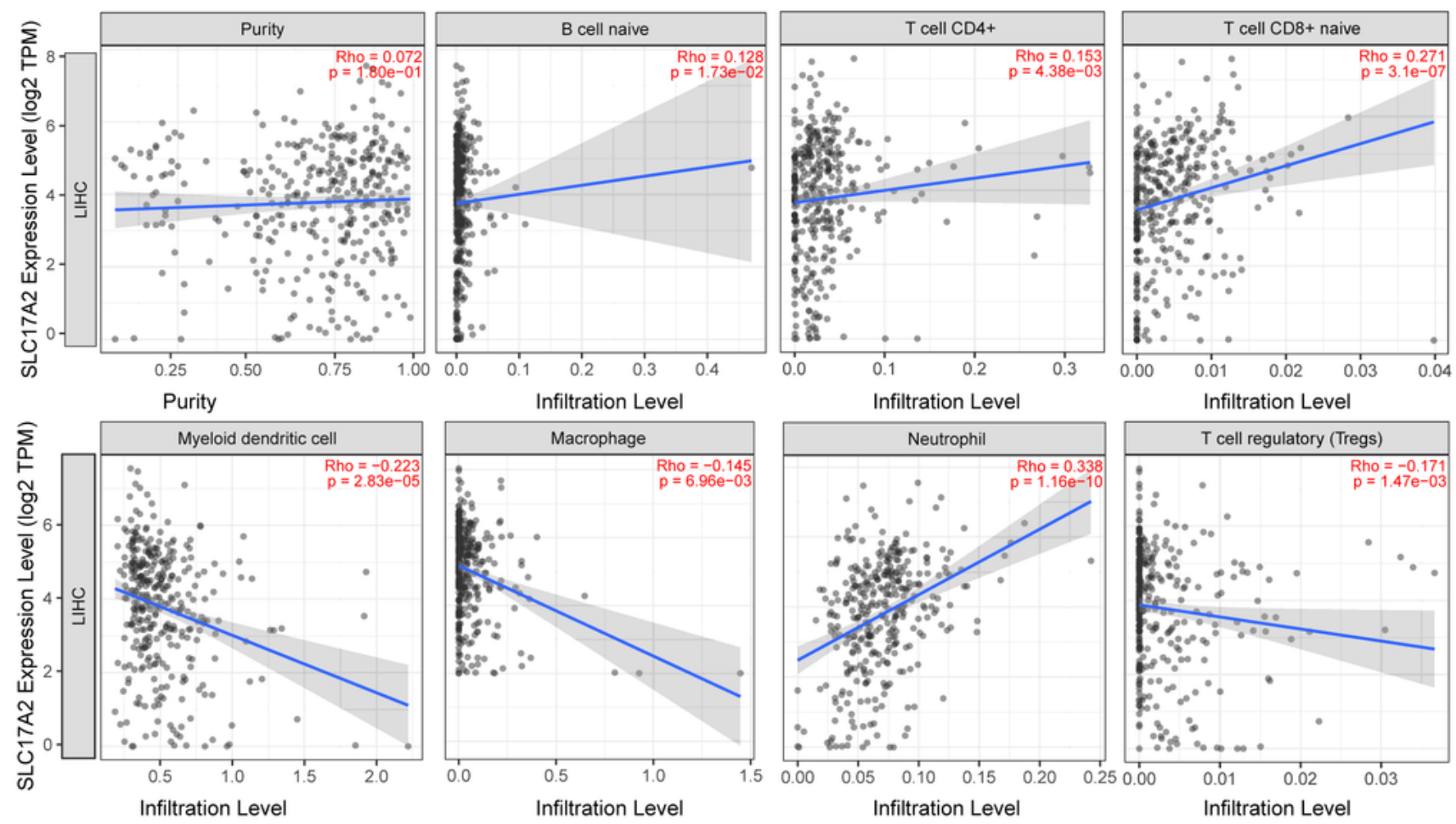

B
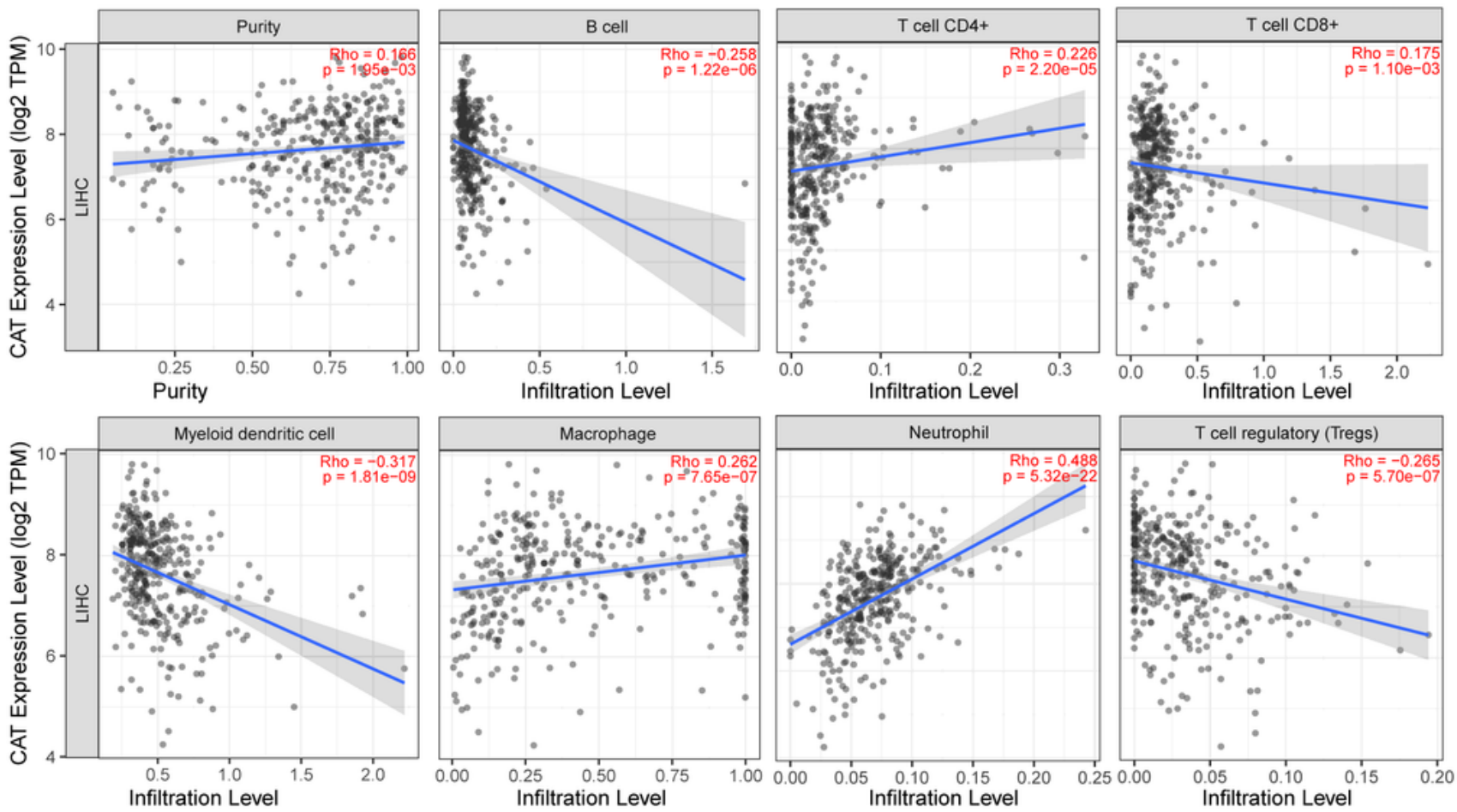

Figure 8

Correlation analysis between SLC17A2 and CAT expression and seven types of infiltrating immune cells in HCC estimated from the TIMER by Spearman's test. The abscissa is the infiltration level of immunocyte, and the ordinate is the gene expression level. A. Relationship between SLC17A2 and immune infiltration. B. Relationship between CAT and immune infiltration. 


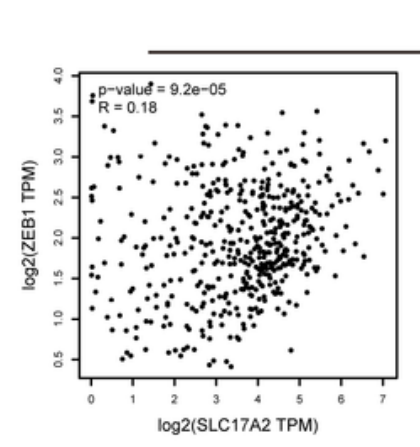

CD8+T cell
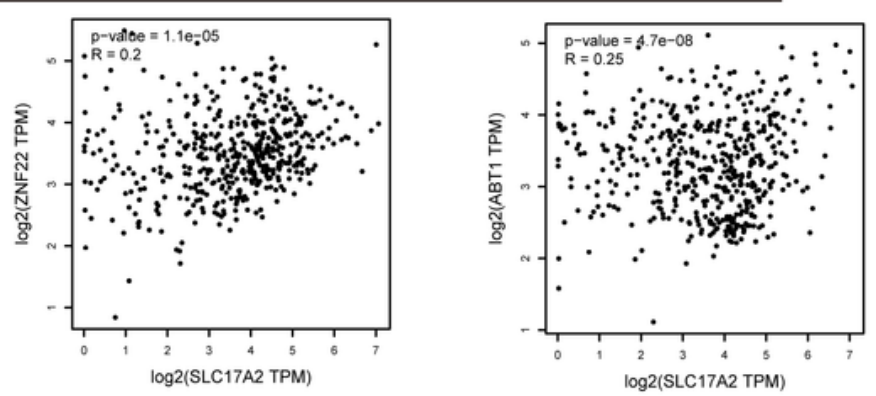

Th2
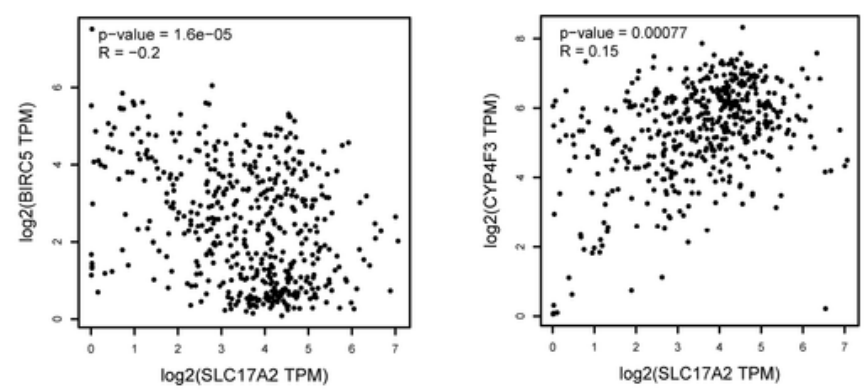

Neutrophils

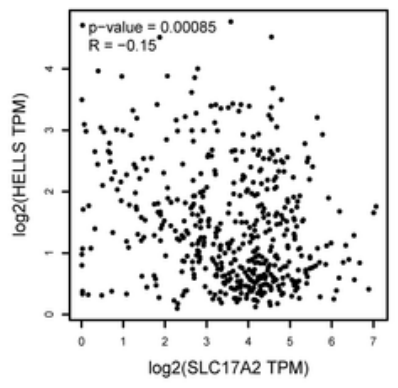

NK cell
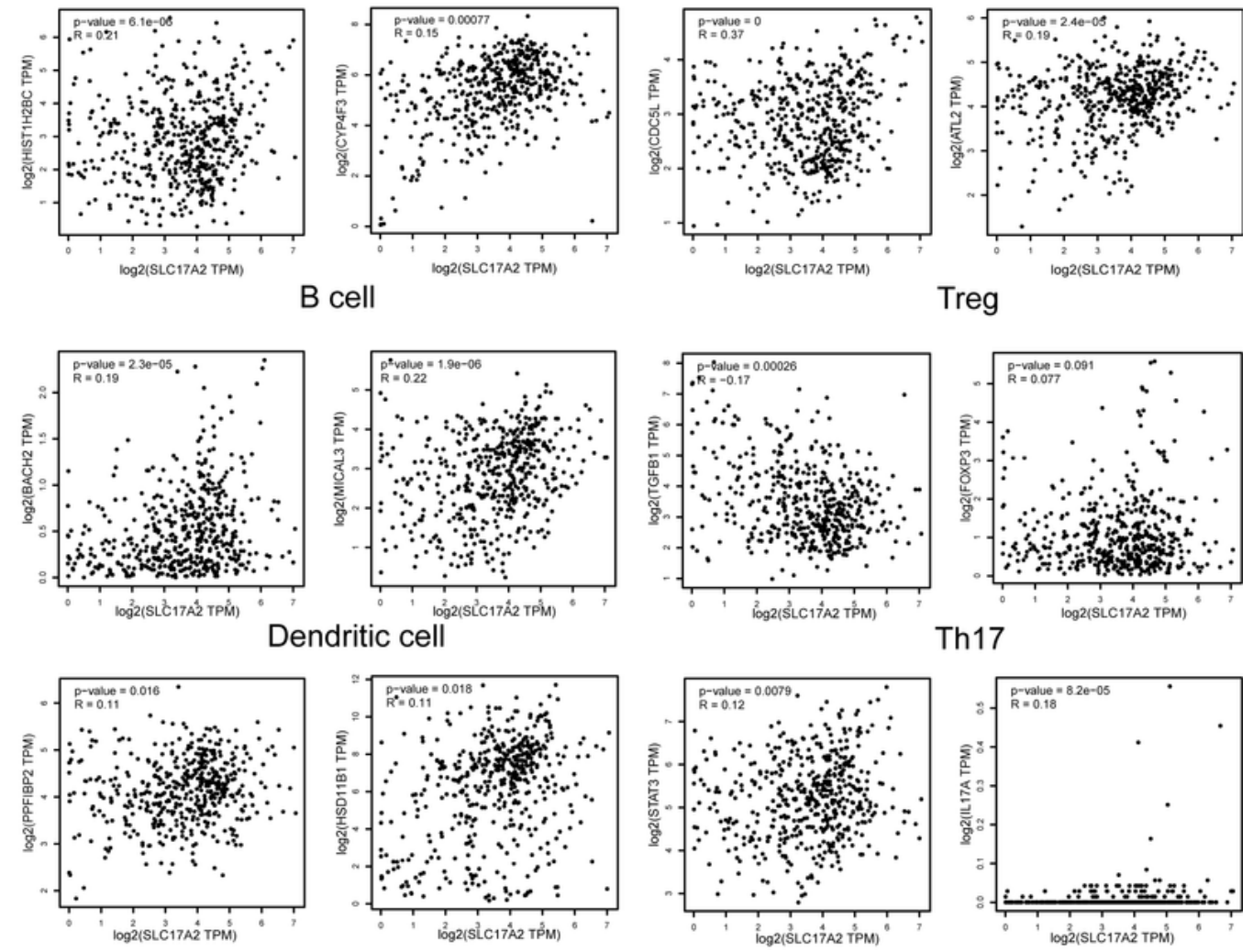

Th17

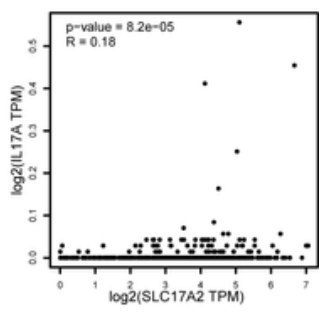

Figure 9

The correlation between the immune cell markers and SLC17A2 expression. 